\title{
Planning of Electroporation-Based Treatments Using Web-Based Treatment-Planning Software
}

\author{
Denis Pavliha $\cdot$ Bor Kos $\cdot$ Marija Marčan $\cdot$ \\ Anže Županič • Gregor Serša $\cdot$ Damijan Miklavčič
}

Received: 10 January 2013/Accepted: 31 May 2013/Published online: 19 June 2013

(c) Springer Science+Business Media New York 2013

\begin{abstract}
Electroporation-based treatment combining high-voltage electric pulses and poorly permanent cytotoxic drugs, i.e., electrochemotherapy (ECT), is currently used for treating superficial tumor nodules by following standard operating procedures. Besides ECT, another electroporation-based treatment, nonthermal irreversible electroporation (N-TIRE), is also efficient at ablating deepseated tumors. To perform ECT or N-TIRE of deep-seated tumors, following standard operating procedures is not sufficient and patient-specific treatment planning is required for successful treatment. Treatment planning is required because of the use of individual long-needle electrodes and the diverse shape, size and location of deepseated tumors. Many institutions that already perform ECT of superficial metastases could benefit from treatmentplanning software that would enable the preparation of patient-specific treatment plans. To this end, we have developed a Web-based treatment-planning software for planning electroporation-based treatments that does not require prior engineering knowledge from the user (e.g., the clinician). The software includes algorithms for automatic tissue segmentation and, after segmentation, generation of a 3D model of the tissue. The procedure allows the user to define how the electrodes will be inserted. Finally,
\end{abstract}

D. Pavliha · B. Kos · M. Marčan · A. Županič .

D. Miklavčič $(\square)$

Faculty of Electrical Engineering, University of Ljubljana,

Tržaška c. 25, SI-1000 Ljubljana, Slovenia

e-mail: damijan.miklavcic@fe.uni-lj.si

D. Pavliha

e-mail: denis.pavliha@fe.uni-lj.si

G. Serša

Institute of Oncology Ljubljana, Zaloška c. 2, SI-1000 Ljubljana,

Slovenia electric field distribution is computed, the position of electrodes and the voltage to be applied are optimized using the 3D model and a downloadable treatment plan is made available to the user.

Keywords Electroporation - Electrochemotherapy · Nonthermal irreversible electroporation .

Treatment planning . Telemedicine

\section{Introduction}

Electroporation is the phenomenon that occurs when a cell is exposed to a sufficiently high external electric field (Kotnik et al. 2012). During and after exposure, the plasma membrane is transiently permeabilized, thus allowing the material from outside to enter the cell, which would be otherwise impeded (Kotnik et al. 1997). The phenomenon is considered to be a universal method and platform technology because all types of cells (animal, plant and microorganisms) are affected by electroporation (Miklavčič 2012). In fact, many electroporation-based applications have already been identified and developed, such as electrochemotherapy (ECT) of tumors (Serša and Miklavčič 2008), nonthermal irreversible ablation of tumors (Garcia et al. 2011; Maor et al. 2009), gene therapy (Heller and Heller 2010), food preservation (Toepfl et al. 2007) and others (Daugimont et al. 2010; Gusbeth et al. 2009; Ušaj et al. 2010).

ECT, which is currently the most developed electroporation-based therapy (Mali et al. 2013; Serša et al. 2008), improves chemotherapy outcome by increasing the plasma membrane permeability to cytotoxic drugs with exposure of target cells (i.e., tumor) to a high-strength electric field (Serša and Miklavčič 2008). The electric field is caused by 
high-voltage electric pulses that are delivered to the target tissue (i.e., tumor) using electrodes (Mir et al. 1991). ECT is already used in clinical practice for treating metastases of skin melanoma in more than 100 clinical institutions in Europe (Miklavčič et al. 2012) and has already been introduced to clinical trials for treating deep-seated metastases in the liver (Edhemović et al. 2011), brain (AgerholmLarsen et al. 2011; Linnert et al. 2012; Mahmood and Gehl 2011), bone (Fini et al. 2011) and soft tissue (Neal et al. 2011). While following standard operating procedures (Mir et al. 2006) ensures safe and successful treatment of skin melanoma metastases (Marty et al. 2006), patient-specific treatment planning is nevertheless required for ECT of deep-seated tumors (Pavliha et al. 2012). Namely, deepseated tumors are very diverse in shape, size and location in the body; and long-needle electrodes are used for treating such tumors; therefore, coverage of the whole target tissue (i.e., tumor) with a sufficiently high electric field (which is a prerequisite for successful ECT) (Miklavčič et al. 1998, 2006) can currently only be assured by means of numerical modeling of electric field distribution. Electric field calculations based on real input data are performed, and imageguided insertion of electrodes is used (Kos et al. 2010; Miklavčič et al. 2010).

Similar to ECT, nonthermal irreversible electroporation (N-TIRE) is another electroporation-based application that is used for ablation of pathological tissue (i.e., tumor) using electrodes (Davalos and Rubinsky 2008; Garcia et al. 2011; Županič and Miklavčič 2009). N-TIRE performs tissue ablation using electric field strengths above values used for ECT and/or with a greater number of electric pulses and, therefore, destroys target tissue without the use of chemotherapeutic drugs or heat (Davalos and Rubinsky 2008). $\mathrm{N}$-TIRE has been in use for treating, e.g., brain metastases (Garcia et al. 2011) and soft-tissue sarcoma (Neal et al. 2011) and would also benefit from patient-specific treatment planning (Golberg and Rubinsky 2012). Although N-TIRE is similar to ECT, it also requires calculations of temperature increase to be included in treatment planning (Županič and Miklavčič 2011).

Patient-specific treatment planning of electroporationbased treatments such as ECT and N-TIRE is based on medical images of the patient, which are used to generate a three-dimensional (3D) model of the target tissue (i.e., tumor) and the organ surrounding the target tissue (Pavliha et al. 2012). Then, the 3D model is used for calculation of the electric field distribution during the electroporationbased treatment (Županič et al. 2012). Since the standard for medical imaging transfer and storage, i.e., digital imaging and communications in medicine (DICOM) (National Electrical Manufacturers Association 2009), represents a common format for storage and transfer of medical images, the images can be transferred over the
Internet and, due to the standard format, the receiver will be able to display them properly. This facilitates treatment planning by developing treatment-planning software as a Web application, which allows remote generation of treatment plans (Olsen et al. 2000). Moreover, the treatment-planning software should be as automated as possible since without treatment-planning software a clinician (i.e., a medical doctor) may otherwise need about $6 \mathrm{~h}$ to manually perform segmentation of a patient (Paulides et al. 2010). The advance from treating skin metastases to treating deep-seated tumors would be facilitated by userfriendly treatment-planning software that would allow clinicians to prepare patient-specific treatment plans without the need of an engineer or a medical physicist or specialized engineering knowledge (Pavliha et al. 2012). Here, we describe implementation of a treatment-planning tool including developed methods for image segmentation, model building and determination of electroporation as a Web-based application for treatment planning of electroporation-based therapies such as ECT and N-TIRE.

\section{Materials and Methods}

\section{Web-Based Graphical User Interface}

The functioning of the Web-based treatment-planning software for electroporation-based treatments is presented in Fig. 1.

The treatment-planning procedure consists of the following subprocedures: upload of medical images (Fig. 1a), selection of series used for planning (Fig. 1b), generation of a 3D model (which is done by preprocessing, segmentation and postprocessing of the images; Fig. 1c), 3D model validation (Fig. 1d), manual correction of segmentation (if decided by the user that it is required, Fig. 1e), virtual insertion of electrodes into the model (Fig. 1f), calculation of electric field distribution (with optimization of parameters: voltages and electrode positions; Fig. 1g) and downloading of the treatment plan (Fig. 1h). In case the images have been previously segmented (Fig. 1i), steps c-e are not executed and pregenerated segments are loaded instead.

In order to develop easy-to-use treatment-planning software, user interaction (i.e., the number of parameters, events or actions the user needs to execute or monitor) should be minimal (Heymann and Degani 2007). Therefore, the most demanding subprocedures (i.e., generation of the 3D model and calculation of the electric field distribution; Fig. 1c, g, respectively) are developed so that they do not require user interaction. Namely, the subprocedures of 3D model generation and electric field distribution calculation require engineering knowledge, such as medical image processing (Birkfellner 2010) and numerical 


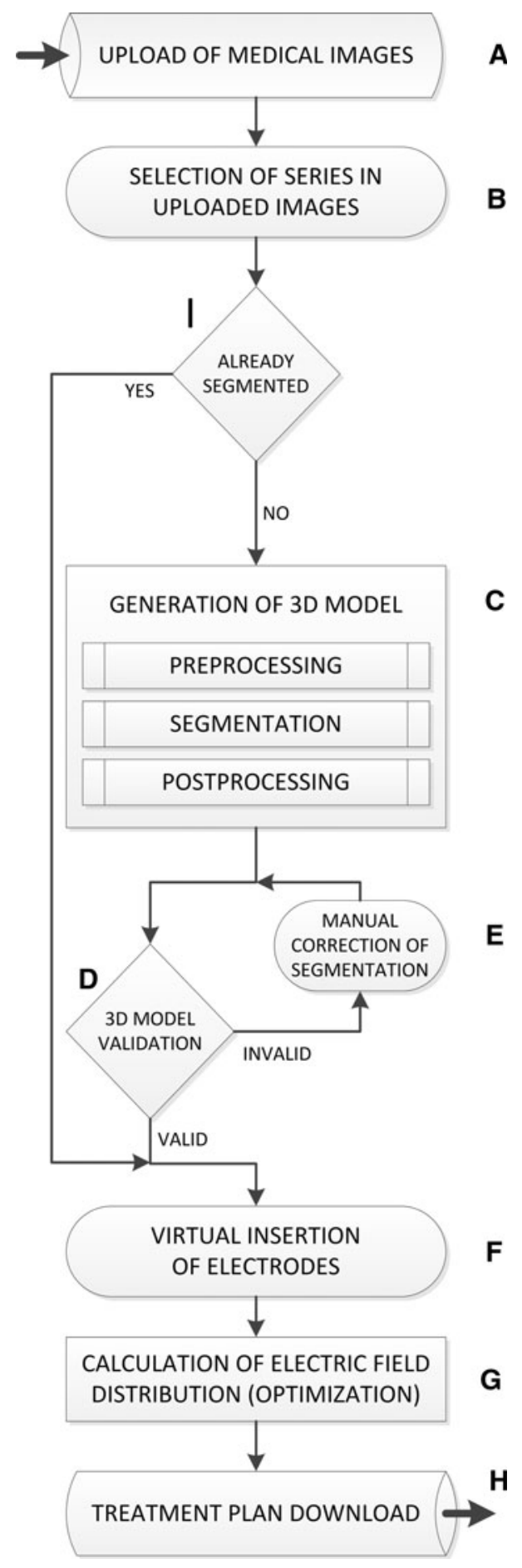

Fig. 1 Flow diagram of Web-based treatment planning for electroporation-based treatments

modeling based on the finite element method in order to perform them. Since users of Web-based treatment-planning software for electroporation-based treatments are expected to be clinicians, all subprocedures are presented in a nonengineering way so that users are able to generate treatment plans effortlessly.
First, the user is required to upload the medical images of the patient (Fig. 1a) by selecting the DICOM files that contain image slices of the body area with the target tissue (i.e., one or more metastases). After uploading the medical images, the treatment-planning software automatically examines them by reading metadata of the DICOM files (i.e., the DICOM header). Parameters SeriesNumber, SeriesDescription and SliceLocation are read from the header; then, the images are grouped by the series they belong to (i.e., SeriesNumber parameter) and labeled using the original series name (i.e., SeriesDescription parameter). Within each group, the images are sorted according to their spatial location (i.e., according to their $Z$ index, i.e., SliceLocation parameter). Afterward, one median image from each group (i.e., each detected series) is presented to the user, who, finally, by clicking on the corresponding series' image, selects which series will be used for planning the electroporation-based treatment (Fig. 1b).

\section{Automatic Segmentation of Medical Images}

Web-based treatment planning for electroporation-based treatments is based on algorithms for automatic segmentation of medical images (Fig. 1c). First, the selected series (Fig. 1b) of the uploaded medical images (Fig. 1a) is preprocessed; all the images (slices) are first transformed using a sigmoid function, described in Eq. (1).

output $=\frac{\text { output_range }}{1+e^{-\frac{4 \text { input-WC }}{\mathrm{WW}}}}$

Parameters window center (WC) and window width (WW) are part of the volume-of-interest metadata located in the DICOM header of each image. The output_range parameter defines the maximum value of the preprocessed image. Parameters input and output are the source and preprocessed data, respectively. All the parameters' values are bits. After transforming each slice using the transformation from Eq. (1), the slices are debiased using a publicly available inhomogeneity correction algorithm (Zheng et al. 2009). Then, each slice is filtered using an average and a gaussian blur filter $(\sigma=3)$, both with window sizes of $3 \times 3$ pixels. Finally, a fixed-value sigmoid transformation ( $\mathrm{WC}=20,000$, $\mathrm{WW}=100$, output_range $=2^{16}$ ) is applied to each slice in order to ensure appropriate intensity distribution, which is necessary for segmentation; fixed values were defined empirically using real-case data.

After preprocessing, image segmentation is performed. Currently, three possible liver segmentation methods are implemented: region growing, adaptive threshold and active contours (i.e., snakes) algorithms. Region growing is a semiautomatic algorithm (because it requires the user to place an initial seed, i.e., to click on the liver on a single 
slice), while adaptive threshold and active contours are automatic algorithms that generate 3D liver models without user interaction. Three liver segmentation algorithms have been implemented because different segmentation algorithms provide different results (De Pasquale and Stander 2009) and user requirements on how the 3D model is constructed may vary. After the liver is segmented, segmentation of tumors and vessels is executed. Algorithms for segmentation of tumors and vessels combine intensitybased and morphological characteristics of given objects to identify them in source images. The basis of the algorithms is a multiscale filter, described in Frangi et al. (1998), which detects local second-order structures based on the relationship between their eigenvalues. The filter distinguishes between linear (or cylindrical in 3D) and round (or spherical in 3D) structures, which can be used for detection of vessels and tumors, respectively. Results of filtering are two image layers: the first includes enhanced tumors, while the other includes enhanced vessel structures. After filtering, the resulting enhanced image layers of tumors and vessels are thresholded using a method based on maximization of entropy (Kapur et al. 1985).

Additionally, region growing is performed in 3D based on thresholding results in order to collect neighboring voxels with intensity values within and below the threshold as these voxels are also part of the segmented structure (Beichel et al. 2004). Region growing is performed iteratively with lowering of the threshold by a factor of 0.001 in each step. The procedure stops once no new voxels have been added in an iteration step. Segmentation of tumors and vessels is performed based on segmentation of the organ that includes them (i.e., the liver) since the segmented target organ is used as a mask for tumor and vessel segmentation. Therefore, tumors are detected if they are in or on the boundary of the organ.

When the segmentation is finished, the user has to validate the generated 3D model (Fig. 1d): segmented layers with the liver, tumor and vessels are presented and manual correction of the generated segments is possible for each slice by dragging the overlaid contours of the generated segments to the desired positions (Fig. 1e). In the final stage, the electrodes are inserted into the 3D model (Fig. 1f). For each identified tumor, a selection of basic electrode configurations is presented to the user. The electrodes are positioned relative to the center of gravity of the tumor at the appropriate depth (Kos et al. 2010) and are parallel to each other. Depending on the planned direction of access, the user can change the direction of insertion of the electrode array, number of electrodes, rotation of the outer electrodes with respect to the electrodes inserted in the tumors, distance between electrodes as well as basic electrode configuration and number of electrodes. When initial positions of all electrode arrays are defined, electric field distribution calculation and optimization of electrode voltage and position are performed.

\section{Electric Field Distribution Calculation and Optimization}

The electric field distribution is the most important predictor of electroporation (Miklavčič et al. 1998, 2006). The electric field distribution can be determined by solving the Laplace equation for electric potential $(V)$ in the static case, described in Eq. (2).

$-\nabla \cdot(\sigma \cdot \nabla V)=0$

Comsol Multiphysics (Comsol, Stockholm, Sweden) is used with the connection to Matlab (Mathworks, Nantick, MA) to automatically build the patient-specific model by using the segmented images for setting location-specific electric properties (Aström et al. 2009), including the electrodes, and solving the electric field distribution. Briefly, the automatic process (Fig. 1g) proceeds as follows: the conductivity of the region of interest is set as a location-based function. The electrodes are then inserted as conductive cylinders of appropriate dimensions (Fig. 1f), and boundary conditions are set. When electrodes are active (i.e., potential is present on them), they are considered as fixed potential, while the nonactive electrodes are left floating with an undefined potential. The simulations are run with a sequential algorithm for increasing conductivities during pulse application (Pavselj et al. 2005; Šel et al. 2005). In brief, the algorithm for determining conductivity changes is based on sequential updating of the conductivity based on the electric field strength from the previous step. Typically, a sigmoid (Šel et al. 2005) or a discrete step-based, piecewise linear function (Županič et al. 2012) is used, although, based on the most recent data, the smoothed Heaviside function offers the best fit to measured in vivo data (Čorović et al. 2013). After the final iteration of the sequential algorithm, the electric field and current density are extracted and the simulations are repeated for all the electrode pairs.

Conductivity values of the different tissues are taken from the literature (Gabriel et al. 1996; Haemmerich et al. 2009), while the change of conductivity due to electroporation is set to a factor of 3.5 (Cukjati et al. 2007). The magnitude of conductivity change has also recently been confirmed on ex vivo chicken liver using magnetic resonance electrical impendance tomographic imaging (Kranjc et al. 2012). Data for conductivity changes of other tissues are still not available, so the same parameters are used for all tissues, with just the reversible and irreversible thresholds changing based on the data available.

The postprocessing of the computed electric fields and currents is handled by Matlab using built-in Comsol 
functions for extracting the electric fields and integrating the current density. The Matlab-Comsol integration provides easy coupling with optimization algorithms, which have access to the output of the models, as well as the ability to subsequently modify electrode positions and applied voltages (Županič et al. 2008, 2012).

The electric field distribution is optimized using a custom-written genetic algorithm, with the entry direction of electrodes specified as an input parameter. The relative electrode positions are optimized, along with the applied voltages (Županič et al. 2008). The constraints of the electric pulse generator (electroporator) are also taken into account. The resulting treatment plans have also been evaluated for robustness to variations in electrode positions and voltages (Kos et al. 2010). The computation of temperature rise can also be included if treatment plans for N-TIRE are desired (Županič and Miklavčič 2011). Finally, the results of treatment planning can be downloaded as a portable document format (PDF) file (Fig. 1h).

\section{Results}

Treatment-planning software for electroporation-based treatments is developed as a Web application. The front-end uses Hyper-text MarkupLanguage 5(HTML5) and JavaScript(JS) forcontentgenerationanduserinteraction, whiletheback-endis a Matlab application invoked by the front-end using Asynchronous JS and XML (AJAX) and a PHP hyper-text preprocessor (The PHP Group, 2001-2012). Three-dimensional visualization is performed using $\mathrm{X}$ Toolkit (The $\mathrm{X}$ Toolkit Developers, 2012, www.goXTK.com), which is a Web GraphicsLibrary-basedtoolkitforscientificvisualization. The graphical user interface (GUI) of the treatment-planning software for electroporation-based treatments is presented in Fig. 2,wherethreeexamplescreensareshown:theinitialscreen (Fig. 2a), the interface for series selection (Fig 2b, procedure from Fig. 1b) and an example generated 3D model of the liver with vessels and an identified tumor (Fig. 2c).

While the GUI is rendered at the client side (i.e., in the user's Web browser), all the processing that involves 3D model generation (Fig. 1c) and electric field distribution calculations (Fig. 1f) is executed on the treatment-planning server; therefore, the user's computer is not overloaded by the functioning of the treatment-planning software (Fig. 3).

\section{Discussion}

Here, we present the possibility of generating treatment plans for electroporation-based treatments remotely (Olsen et al. 2000), i.e., using Web-based treatment-planning software and without prior engineering knowledge or the help of an engineer. The software is based on algorithms for automatic segmentation; currently, three different algorithms for liver segmentation are implemented. Because the software is developed as modular, future inclusion of additional segmentation modules is possible with instantaneous deployment. Namely, the developed software provides a modular framework that allows inclusion of additional segmentation algorithms; therefore, modules for segmentation of other tissue (e.g., bone, softtissue sarcoma) may be included in the Web application. Computation of electric fields in additional tissues is also possible since segmented images are used as postprocessing and electric property masks in the simulation step. One of the largest limitations is the availability of data on electrical properties of tissues and data on changes of electric conductivity during pulse application, which, however, can be obtained through an iterative procedure (i.e., modeling and measurements) or by inverse analysis (Čorović et al. 2013). However, this can be circumvented to some degree by approximating the values using data from the literature and keeping the relative conductivity of the target tissue versus surrounding tissue high, which gives a conservative estimation of field distribution (Kos et al. 2010).

Currently, the software cannot perform real-time (i.e., online) calculations because both image segmentation and numerical calculations of the electric field distribution are time-consuming processing operations. Nevertheless, realtime visualization of the electric field distribution could contribute to a better understanding of the electrode positioning during the virtual placement procedure. To this end, a software application, ApiVizTEP, which performs simplified, rough calculation of the electric field distribution for visualization purposes, has already been developed (Mahnič-Kalamiza et al. 2012). ApiVizTEP is open-source and can be downloaded from http://sourceforge.net/ projects/apiviztep/. In the future, an integration of ApiVizTEP into the Web-based treatment-planning software could be considered.

The presented software allows loading cases that were previously segmented (Fig. 1i), which is important for historical evaluation of patients who were already treated. The possibility of loading presegmented cases brings two major benefits: (1) the clinicians can perform historical evaluation of cases who were previously treated with ECT or N-TIRE and evaluate if there is a correlation between the calculated coverage of the tumor with the electric field and (2) the software is able to perform calculations of the electroporation-based treatment regardless of the tissue/ organ that is subjected to treatment provided that segmentation data are available. If the segmentation is performed by radiologists, the presented treatment-planning 
Fig. 2 Graphical user interface (GUI) of the Web-based treatment-planning software for electroporation-based treatments. Presented are three example screens: initial screen a, interface for series selection b and an example generated 3D model of liver with a tumor and major vessels $\mathbf{c}$

\section{ECTplan}

Welcome
Load Images
Planning
Validation
Save
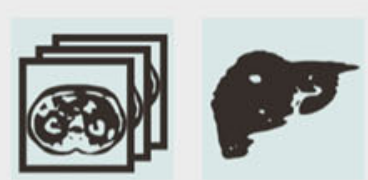

Begin with loading the images by clicking on the Load Images menu item.

University of Ljubljana, Slovenia (EU)

D. Pavliha, B. Kos, M. Marčan, A. Županič, G. Serša, D. Miklavčič

\section{ECTplan}

\section{Welcome \\ Load Images \\ Planning \\ Validation}
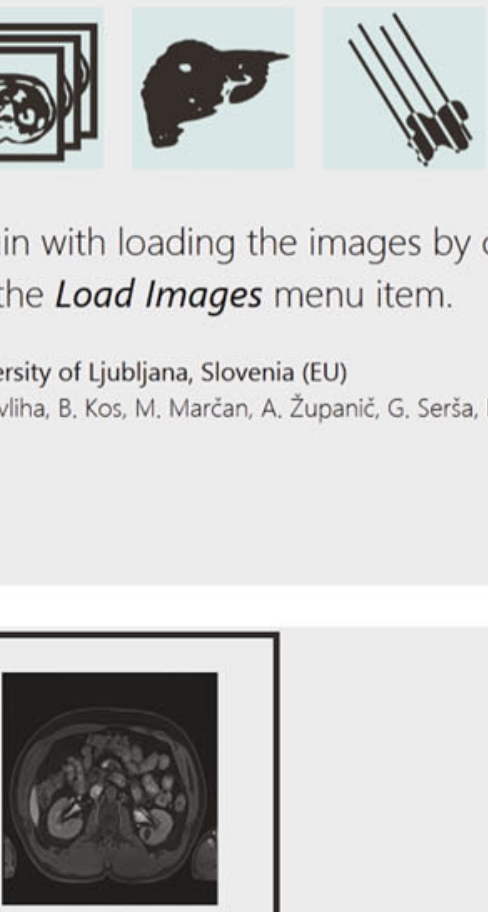

A

Welcome to Electrochemotherapy Treatment Planning!

Save

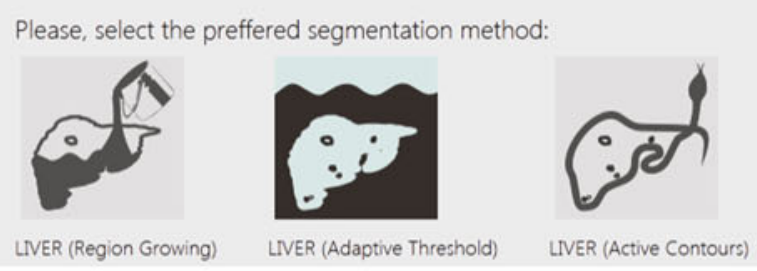

\section{ECTplan}

C
The 3D model is successfully generated:

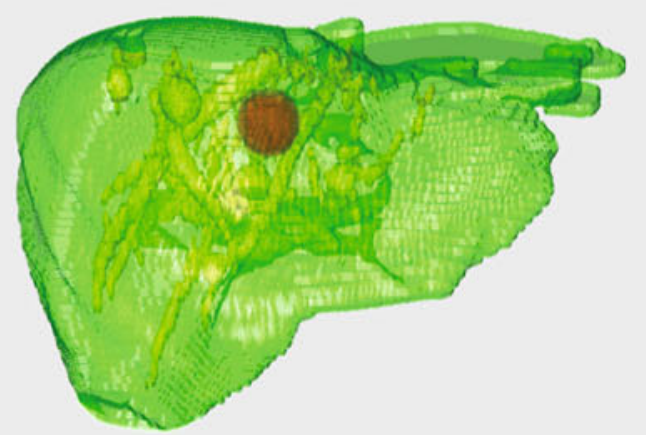

You may now proceed to validation. 
Fig. 3 Example of generated treatment plan, showing an electroporation cross section overlaid with the original patient image a, cumulative coverage curves showing the volume fraction of tumor treated above a certain electric field b and electrode pair contributions indicating the contribution of each individual electrode pair $\mathbf{c}$
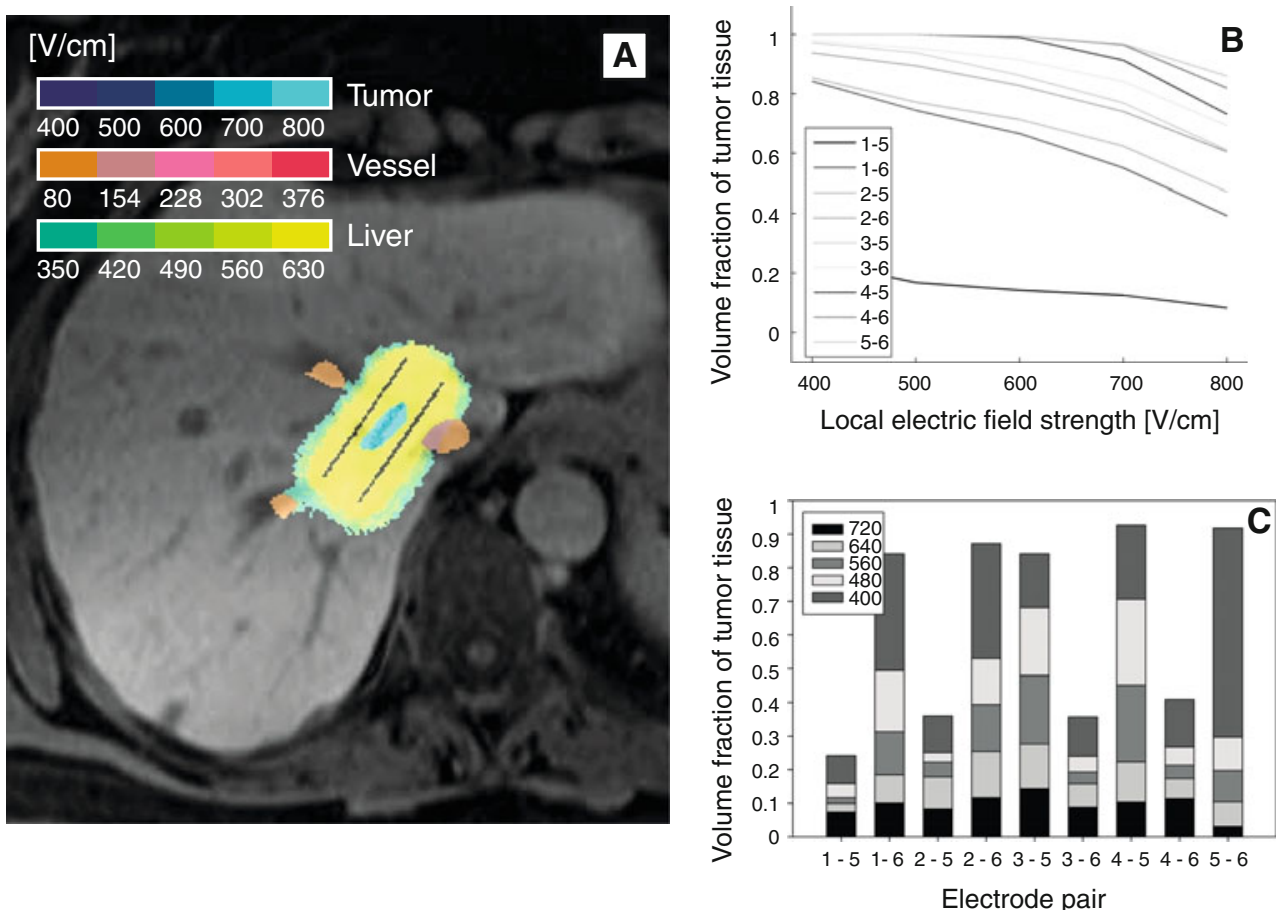

Electrode pair software allows calculations and optimization of the electric field distribution in the segmented tissue/organ. In Fig. 4, an example of model geometry representing the prostate is shown. The model was previously segmented by a radiologist, segments were then imported into the treatment-planning software and, finally, a treatment plan for N-TIRE was calculated.

Algorithms for segmentation of tumor and vessel structures need to be robust, especially with respect to different imaging modalities of source images. Based on our experience with real-case data, MRIs may include representations of tumors and vessels with very similar intensities. Therefore, intensity-based segmentation methods such as thresholding cannot successfully separate tumors from vessels in MRIs. This drawback can be overcome by incorporating structural information into the segmentation algorithm: by observing the structures in three dimensions through all slices, it is possible to separate blob-like structures which might be tumors from line structures which are typical for vessels. After execution of the segmentation algorithm which detects blobs, an additional step is required to define whether the identified structures are tumors or healthy tissue. Such verification is performed in the validation step (Fig. 1d), where segmentation of all the tissues is manually validated by the user, who has the possibility of accepting the segmentation results or correcting them manually (i.e., each slice) before continuing with virtual electrode insertion.
Finally, the results of treatment planning that are produced by electric field distribution optimization need to be presented in understandable and human-readable form. We have previously developed several novel visualization methods, allowing visualization of the contribution of each electrode pair toward the total treatment and visualization of the coverage of tissues above a certain electric field (as well as visualization of predicted electroporation) overlaid on the original medical images. In the future, the treatment plan can be transferred directly to electroporation devices (e.g., electroporators or devices for robot-assisted surgery), which will facilitate electroporation-based treatments even more.

An important concern involves the implementation of the software among end-users, which is related to liability when preparing treatment plans using the software. Since in radiotherapy, a well-established treatment plan we followed (Pavliha et al. 2012), the clinician is always responsible for final validation of the treatment plan, the validation of image segmentation for electroporation-based treatment planning is an imperative step. Also, use of the treatment-planning software will be introduced in stages: first, we formed a closed group of beta testers who were offered the possibility to evaluate the functioning of our software. The information they provided will serve us for improving the functioning, and afterward the software will be made available for research purposes. A final step will be certifying the software for clinical use. 
Fig. 4 Excerpt from a prepared treatment plan for N-TIRE of prostate cancer. a Electrode pair contribution, b model with three needle electrodes, c cumulative coverage curves showing that the whole tumor is covered with fields in excess of $700 \mathrm{~V} / \mathrm{cm}$ and $>95 \%$ of the tumor is covered with fields in excess of $800 \mathrm{~V} / \mathrm{cm}$, d voltages used in the proposed treatment plan a

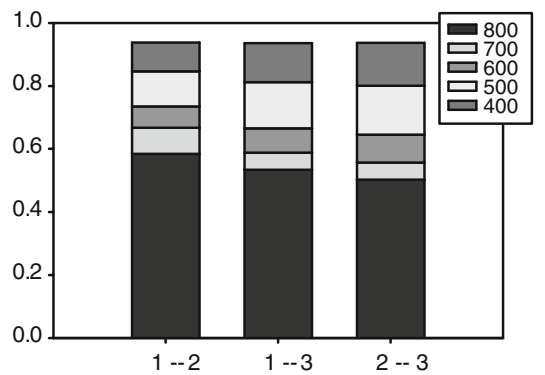

C

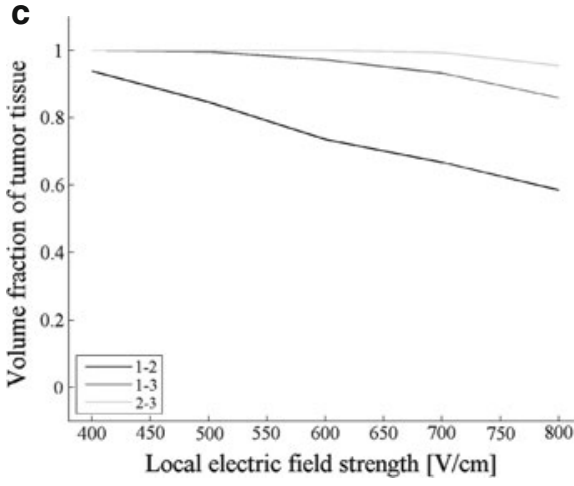

b Rectum

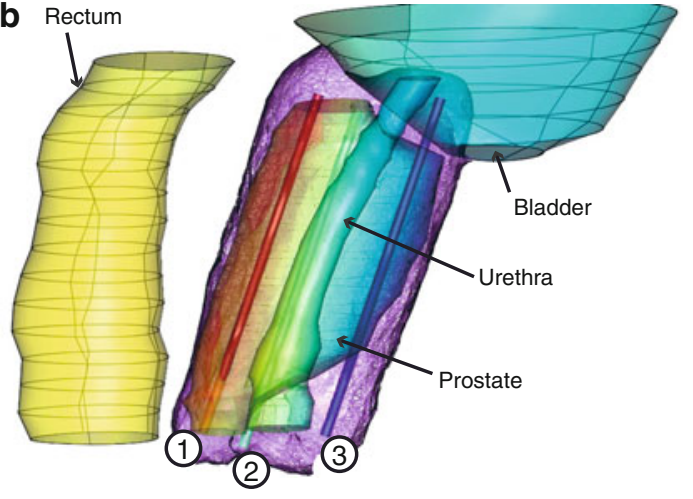

d

\begin{tabular}{cc} 
Electrode pair & Voltage \\
\hline $1-2$ & 2200 \\
$1-3$ & 2500 \\
$2-3$ & 2400
\end{tabular}

\section{Conclusion}

Because a well-defined target (i.e., tumor or other pathological tissue) needs to be determined when planning electroporation-based treatments (such as ECT or N-TIRE) of deep-seated tumors (Pavliha et al. 2012) and distribution of the electric field in biological tissue is the most important predictor of electroporation (Miklavčič et al. 1998, 2006), clinicians and eventually patients can greatly benefit from the Web-based treatment-planning software we have developed. Namely, the software allows generation of electroporation-based treatment plans without prior engineering knowledge and generates 3D models based on patient images that are used for calculation and optimization of the electric field distribution during electroporation. Moreover, it is possible to import previously segmented cases, which allows historical evaluation of patients who were already treated as well as preparation of treatment plans for tissue types which are currently not supported by the automatic segmentation algorithms but could also benefit from Web-based treatment planning. Finally, the treatment plan is presented in a clearly understandable, human-readable form. Therefore, the Web-based treatment-planning software could contribute to the faster advance of clinicians already practicing electroporation-based treatments of superficial tumor nodules toward treating deep-seated tumors, such as bone metastasis and soft-tissue sarcoma (Miklavčič et al. 2012).
Acknowledgement This work was supported by the Slovenian Research Agency. Research was conducted in the scope of the Electroporation in Biology and Medicine, European Associated Laboratory. The authors thank Dr. Robert Hudej from the Institute of Oncology, Ljubljana, for providing data that were used for preparing the treatment plan for N-TIRE of prostate cancer.

\section{References}

Agerholm-Larsen B, Iversen HK, Ibsen P et al (2011) Preclinical validation of electrochemotherapy as an effective treatment for brain tumors. Cancer Res 71:3753-3762

Aström M, Zrinzo LU, Tisch S et al (2009) Method for patientspecific finite element modeling and simulation of deep brain stimulation. Med Biol Eng Comput 47:21-28

Beichel R, Pock T, Janko C et al (2004) Liver segment approximation in CT data for surgical resection planning. SPIE medical imaging '04, vol 5370. SPIE, San Diego, pp 1435-1446

Birkfellner W (2010) Applied medical image processing: a basic course. Taylor and Francis, New York

Čorović S, Lacković I, Šuštaršič P et al (2013) Modeling of electric field distribution in tissues during electroporation. Biomed Eng Online 12:16

Cukjati D, Batiuskaite D, Andre F et al (2007) Real time electroporation control for accurate and safe in vivo non-viral gene therapy. Bioelectrochemistry 70:501-507

Daugimont L, Baron N, Vandermeulen G et al (2010) Hollow microneedle arrays for intradermal drug delivery and DNA electroporation. J Membr Biol 236:117-125

Davalos R, Rubinsky B (2008) Temperature considerations during irreversible electroporation. Int J Heat Mass Transf 51:56175622

De Pasquale F, Stander J (2009) A multi-scale template method for shape detection with bio-medical applications. Pattern Anal Appl 12:179-192 
Edhemović I, Gadžijev EM, Brecelj E et al (2011) Electrochemotherapy: a new technological approach in treatment of metastases in the liver. Technol Cancer Res Treat 10:475-485

Fini M, Tschon M, Alberghini M et al (2011) Cell electroporation in bone tissue. In: Key ST, Gehl J, Lee EW (eds) Clinical aspects of electroporation. Springer, New York, pp 115-127

Frangi A, Niessen W, Vincken K, Viergever M (1998) Multiscale vessel enhancement filtering. In: Wells W, Colchester A, Delp S (eds) Medical image computing and computer-assisted intervention. Springer, Berlin, pp 130-137

Gabriel S, Lau R, Gabriel C (1996) The dielectric properties of biological tissues: II. Measurements in the frequency range $10 \mathrm{~Hz}$ to $20 \mathrm{GHz}$. Phys Med Biol 41:2251-2269

Garcia P, Pancotto T, Rossmeisl J et al (2011) Non-thermal irreversible electroporation (N-TIRE) and adjuvant fractionated radiotherapeutic multimodal therapy for intracranial malignant glioma in a canine patient. Technol Cancer Res Treat 10:73-83

Golberg A, Rubinsky B (2012) Towards electroporation based treatment planning considering electric field induced muscle contractions. Technol Cancer Res Treat 11:189-201

Gusbeth C, Frey W, Volkmann H et al (2009) Pulsed electric field treatment for bacteria reduction and its impact on hospital wastewater. Chemosphere 75:228-233

Haemmerich D, Schutt D, Wright A et al (2009) Electrical conductivity measurement of excised human metastatic liver tumours before and after thermal ablation. Physiol Meas 30: 459-466

Heller L, Heller R (2010) Electroporation gene therapy preclinical and clinical trials for melanoma. Curr Gene Ther 10:312-317

Heymann M, Degani A (2007) Formal analysis and automatic generation of user interfaces: approach, methodology, and an algorithm. Hum Factors 49:311-330

Kapur JN, Sahoo PK, Wong AKC (1985) A new method for graylevel picture thresholding using the entropy of the histogram. Comput Vis Graph Image Process 29:273-285

Kos B, Županič A, Kotnik T et al (2010) Robustness of treatment planning for electrochemotherapy of deep-seated tumors. J Membr Biol 236:147-153

Kotnik T, Bobanovič F, Miklavčič D (1997) Sensitivity of transmembrane voltage induced by applied electric fields-a theoretical analysis. Bioelectrochem Bioenerg 43:285-291

Kotnik T, Kramar P, Pucihar G et al (2012) Cell membrane electroporation. Part 1: The phenomenon. IEEE Electr Insul Mag 28:14-23

Kranjc M, Bajd F, Sersa I et al (2012) Ex vivo and in silico feasibility study of monitoring electric field distribution in tissue during electroporation based treatments. PLoS One 7:e45737

Linnert M, Iversen H, Gehl J (2012) Multiple brain metastasescurrent management and perspectives for treatment with electrochemotherapy. Radiol Oncol 2012:1-8

Mahmood F, Gehl J (2011) Optimizing clinical performance and geometrical robustness of a new electrode device for intracranial tumor electroporation. Bioelectrochemistry 81:10-16

Mahnič-Kalamiza S, Kotnik T, Miklavčič D (2012) Educational application for visualization and analysis of electric field strength in multiple electrode electroporation. BMC Med Educ $12: 102$

Mali B, Jarm T, Snoj M et al (2013) Antitumor effectiveness of electrochemotherapy: a systematic review and meta-analysis. Eur J Surg Oncol 39:4-16

Maor E, Ivorra A, Rubinsky B (2009) Nonthermal irreversible electroporation: novel technology for vascular smooth muscle cells ablation. PLoS One. doi:10.1371/journal.pone.0004757

Marty M, Serša G, Garbay J et al (2006) Electrochemotherapy-an easy, highly effective and safe treatment of cutaneous and subcutaneous metastases: results of ESOPE (European Standard
Operating Procedures of Electrochemotherapy) study. Eur J Cancer 4:3-13

Miklavčič D (2012) Network for development of electroporationbased technologies and treatments: COST TD1104. J Membr Biol 245:591-598

Miklavčič D, Beravs K, Šemrov D et al (1998) The importance of electric field distribution for effective in vivo electroporation of tissues. Biophys J 74:2152-2158

Miklavčič D, Čorović S, Pucihar G, Pavšelj N (2006) Importance of tumour coverage by sufficiently high local electric field for effective electrochemotherapy. EJC 4:45-51

Miklavčič D, Snoj M, Županič A et al (2010) Towards treatment planning and treatment of deep-seated solid tumors by electrochemotherapy. Biomed Eng Online 9:10

Miklavčič D, Serša G, Brecelj E et al (2012) Electrochemotherapy: technological advancements for efficient electroporation-based treatment of internal tumors. Med Biol Eng Comput 50: $1213-1225$

Mir LM, Orlowski S, Belehradek J, Paoletti C (1991) Elecrochemotherapy potentiation of antitumor effect of bleomycin by local electric pulses. Eur J Cancer 27:68-72

Mir L, Gehl J, Sersa G et al (2006) Standard operating procedures of the electrochemotherapy: instructions for the use of bleomycin or cisplatin administered either systemically or locally and electric pulses delivered by the Cliniporator ${ }^{\mathrm{TM}}$ by means of invasive or non-invasive electrodes. EJC Suppl 4:14-25

National Electrical Manufacturers Association (2009) Digital imaging and communications in medicine (DICOM)

Neal RE, Rossmeisl JH Jr, Garcia PA et al (2011) Successful treatment of a large soft tissue sarcoma with irreversible electroporation. J Clin Oncol 29:E372-E377

Olsen D, Bruland O, Davis B (2000) Telemedicine in radiotherapy treatment planning: requirements and applications. Radiother Oncol 54:255-259

Paulides MM, Bakker JF, Linthorst M et al (2010) The clinical feasibility of deep hyperthermia treatment in the head and neck: new challenges for positioning and temperature measurement. Phys Med Biol 55:2465

Pavliha D, Kos B, Županič A et al (2012) Patient-specific treatment planning of electrochemotherapy: procedure design and possible pitfalls. Bioelectrochemistry 87:265-273

Pavselj N, Bregar Z, Cukjati D et al (2005) The course of tissue permeabilization studied on a mathematical model of a subcutaneous tumor in small animals. IEEE Trans Biomed Eng 52: 1373-1381

Šel D, Cukjati D, Batiuskaite D et al (2005) Sequential finite element model of tissue electropermeabilization. IEEE Trans Biomed Eng 52:816-827

Serša G, Miklavčič D (2008) Electrochemotherapy of tumors. J Visualized Experiments 22

Serša G, Miklavčič D, Čemažar M et al (2008) Electrochemotherapy in treatment of tumours. Eur J Surg Oncol 34:232-240

Toepfl S, Heinz V, Knorr D (2007) High intensity pulsed electric fields applied for food preservation. Chem Eng Proc 46:537-546

Ušaj M, Trontelj K, Miklavčič D, Kandušer M (2010) Cell-cell electrofusion: optimization of electric field amplitude and hypotonic treatment for mouse melanoma (B16-F1) and Chinese hamster ovary $(\mathrm{CHO})$ cells. J Membr Biol 236:107-116

Zheng Y, Grossman M, Awate SP, Gee JC (2009) Automatic correction of intensity nonuniformity from sparseness of gradient distribution in medical images. Proceedings of the 12th international conference on medical image computing and computer-assisted intervention: part II. Springer, Berlin, pp 852-859

Županič A, Miklavčič D (2009) Optimization and numerical modeling in irreversible electroporation treatment planning. In: 
Rubinsky B (ed) Irreversible electroporation. Springer, Berlin, pp 203-222

Županič A, Miklavčič D (2011) Tissue heating during tumor ablation with irreversible electroporation. Electrotech Rev 78:42-47

Županič A, Čorović S, Miklavčič D (2008) Optimization of electrode position and electric pulse amplitude in electrochemotherapy. Radiol Oncol 42:93-101
Županič A, Kos B, Miklavčič D (2012) Treatment planning of electroporation-based medical interventions: electrochemotherapy, gene electrotransfer and irreversible electroporation. Phys Med Biol 57:5425-5440 